\title{
2HybridTools, a handy software to facilitate clone identification and mutation mapping from yeast two-hybrid screening
}

\author{
Pierre Cauchy ${ }^{\text {Corresp., 1, 2,3,4,5 }}$, Brigitte Kahn-Perlès ${ }^{3,4,5}$, Pierre Ferrier ${ }^{2,5}$, Jean Imbert ${ }^{3,4,5}$, Patrick Lécine ${ }^{\text {Corresp. } 4,5,6}$ \\ 1 Max Planck Institute for Immunobiology and Epigenetics, Freiburg, Germany \\ 2 Centre d'Immunologie de Marseille-Luminy, Inserm U1104, CNRS UMR7280, Marseille, France \\ 3 TAGC, Inserm U1090, Marseille, France \\ 4 Centre de Recherche en Cancérologie de Marseille, Inserm UMR1068, CNRS UMR7256, Marseille, France \\ 5 Université de la Mediterranée (Aix-Marseille II), Marseille, France \\ 6 Vaccine Thematic Unit, BIOASTER, Lyon, France \\ Corresponding Authors: Pierre Cauchy, Patrick Lécine \\ Email address: cauchy@ie-freiburg.mpg.de, patrick.lecine@bioaster.org
}

Yeast Two-Hybrid $(\mathrm{Y} 2 \mathrm{H})$ and reverse Two-Hybrid ( $\mathrm{RY} 2 \mathrm{H})$ are powerful protein-protein interaction screening methods that rely on the interaction of bait and prey proteins fused to DNA binding (DB) and activation domains (AD), respectively. $\mathrm{Y} 2 \mathrm{H}$ allows identification of protein interaction partners using screening libraries, while $\mathrm{RY} 2 \mathrm{H}$ is used to determine residues critical to a given protein-protein interaction by exploiting site-directed mutagenesis. Currently, both these techniques still rely on sequencing of positive clones using conventional Sanger sequencing. For $\mathrm{Y} 2 \mathrm{H}$, a screen can yield several positives; the identification of such clones is further complicated by the fact that sequencing products usually contain vector sequence. For $\mathrm{RY} 2 \mathrm{H}$, obtaining a complete sequence is required to identify the full range of residues involved in protein-protein interactions. However, with Sanger sequencing limited to 500-800 nucleotides, sequencing is usually carried from both ends for clones greater than this length. Analysis of such RY2H data thus requires assembly of sequencing products combined with trimming of vector sequences and of lowquality bases at the beginning and ends of sequencing products. Further, $\mathrm{RY} 2 \mathrm{H}$ analysis requires collation of mutations that abrogate a $D B / A D$ interaction. Here, we present 2 HybridTools, a Java program with a user-friendly interface that allows addressing all these issues inherent to both $\mathrm{Y} 2 \mathrm{H}$ and $\mathrm{RY} 2 \mathrm{H}$. Specifically, for $\mathrm{Y} 2 \mathrm{H}, 2 \mathrm{HybridTools}$ enables automated identification of positive clones, while for $\mathrm{RY} 2 \mathrm{H}, 2 \mathrm{HybridTools}$ provides detailed mutation reports as a basis for further investigation of given protein-protein interactions. 
1 2HybridTools, a handy software to facilitate clone identification and mutation mapping from yeast two3 hybrid screening

Pierre Cauchy ${ }^{1-5^{*}}$, Brigitte Kahn-Perlès ${ }^{3-5}$, Pierre Ferrier ${ }^{2,5}$, Jean Imbert ${ }^{3-5}$,Patrick Lécine ${ }^{4-6^{*}}$

${ }^{1}$ Max Planck Institute for Immunobiology and Epigenetics, Freiburg, Germany

${ }^{2}$ Centre d'Immunologie de Marseille-Luminy, Inserm U1104, CNRS UMR7280, Marseille, France;

${ }^{3}$ TAGC, Inserm U1090, Marseille, France;

${ }^{4}$ Centre de Recherche en Cancérologie de Marseille, Inserm UMR1068, CNRS UMR7256, Marseille, France

${ }^{5}$ Université de la Méditerranée (Aix-Marseille II), Marseille, France;

${ }^{6}$ Vaccine Thematic Unit, BIOASTER, Lyon, France

${ }^{*}$ Corresponding Author:

Patrick Lécine

Vaccine Thematic Unit, BIOASTER, 40 Avenue Tony Garnier, 69007 Lyon, France

Email address: patrick.lecine@,bioaster.org

Pierre Cauchy

Max Planck Institute for Immunobiology and Epigenetics, Department of Cellular and Molecular Immunology, Stübeweg 51, 79108 Freiburg im Breisgau, Germany

Email address: cauchy@,ie-freiburg.mpg.de

\section{Abstract}

Yeast Two-Hybrid $(\mathrm{Y} 2 \mathrm{H})$ and reverse Two-Hybrid $(\mathrm{RY} 2 \mathrm{H})$ are powerful protein-protein interaction screening methods that rely on the interaction of bait and prey proteins fused to DNA binding (DB) and activation domains (AD), respectively. $\mathrm{Y} 2 \mathrm{H}$ allows identification of protein interaction partners using screening libraries, while RY2H is used to determine residues critical to a given protein-protein interaction by exploiting site-directed mutagenesis. Currently, both these techniques still rely on sequencing of positive clones using conventional Sanger sequencing. For Y2H, a screen can yield several positives; the identification of such clones is further complicated by the fact that sequencing products usually contain vector sequence. For $\mathrm{RY} 2 \mathrm{H}$, obtaining a complete sequence is required to identify the full range of residues involved in protein-protein interactions. However, with Sanger sequencing limited to 500-800 nucleotides, sequencing is usually carried from both ends for clones greater than this length. Analysis of such $\mathrm{RY} 2 \mathrm{H}$ data thus requires assembly of sequencing products combined with trimming of vector sequences and of low-quality bases at the beginning and ends of sequencing products. Further, $\mathrm{RY} 2 \mathrm{H}$ analysis requires collation of mutations that abrogate a $\mathrm{DB} / \mathrm{AD}$ interaction. Here, we 
present 2 HybridTools, a Java program with a user-friendly interface that allows addressing all these issues inherent to both Y2H and RY2H. Specifically, for Y2H, 2HybridTools enables automated identification of positive clones, while for RY2H, 2HybridTools provides detailed mutation reports as a basis for further investigation of given protein-protein interactions.

\section{Introduction}

Determining the function(s) of all proteins is one of the major challenges in the post genomic area. This long term goal is relevant to fundamental biology as well as translational research such as drug development, since molecular target discovery and validation require an understanding of the function and disease relevance of the proteins. One important step toward the elucidation of their function(s) is to characterize the molecular interacting networks in which they are implicated. In this context, recent modifications of the popular yeast two-hybrid system (Y2H) have been made, enhancing both its stringency and flexibility, resulting in development of new applications such as the reverse yeast two-hybrid system (RY2H) (Bruckner et al. 2009; Vidal \& Legrain 1999).

$\mathrm{Y} 2 \mathrm{H}$ is based on the fact that many sequence-specific transcription factors (TFs) increase the rate of transcription of their target genes by binding to cis-acting regulatoring elements (CREs) and activating RNA-polymerase (Pol) II at the corresponding promoters. The DNA-binding and activating functions are usually located in physically separable domains, which are referred to as the DNA-binding domain (DB) and the activation domain (AD), respectively. It has been shown that coupling them respectively with any paired protein-protein interaction domains, can reconstitute a functional TF by bringing $\mathrm{DB}$ and $\mathrm{AD}$ into close physical proximity (Fields \& Song 1989). Thus, the reconstitution of a functional TF can be summarized as DB-X:AD-Y, where $\mathrm{X}$ and $\mathrm{Y}$ could be essentially any protein domains from any organism. When yeast-growth selection markers such as URA3 or HIS3 (genes involved in uracil and histidine synthesis, respectively) are expressed from a promoter containing DB-binding sites, the DB-X:AD-Y interaction confers a selective advantage. Thus, a few growing yeast colonies can be identified on plates lacking the corresponding amino acid. Such positive selections have been used to identify a great number of specific protein-protein interactions by screening of cDNA libraries (Hamdi \& Colas 2012; James et al. 1996; Parrish et al. 2006).

Conceptually, protein-protein interactions can also be inhibited by the use of cis-acting mutationsin one partner (referred to as interaction-defective alleles, IDAs) or transacting molecules such as dissociating peptides or small molecules. For example, IDAs can be compared with their wild-type counterparts for their ability to functionally complement a knock-out in the corresponding gene or for their ability to function in an expression assay in the relevant cells. In this Reverse version of $\mathrm{Y} 2 \mathrm{H}$, the wild-type DB-X:AD-Y interaction is toxic or lethal for the yeast cells because a toxic marker (e.g. URA3 in presence of 5-fluoroorotic acid,5-FOA) is used as a reporter gene (negative selection). Yeast cells that express $U R A 3$, a gene normally involved in the synthesis of uracil, fail to grow on media containing 5-FOA because the URA3 enzyme transforms 5-FOA into a toxic compound. Thus, DB-X:AD-Y expressing cells are 5-FOA 
sensitive $\left(5-\mathrm{FOA}^{\mathrm{S}}\right)$. In this setting, DB-X:AD-Y dissociation confers a selective growth advantage that can conveniently identify both interaction-defective alleles. Interactions tested in RY2H can stem from known protein-protein interactions, or from ones inferred computationally (Sardina et al. 2018).

Many different systems and variants have been optimized to reduce the number of false positive clones, resulting in an accumulation of relevant biological data, including data from large scale analysis of the human interactome or protein networks involved in human diseases, in viral replication as well as in drug discovery (Hollingsworth 2004; Hughes 2002; Lim et al. 2006; Rual et al. 2005). Consequently, when dealing with large scale analysis coupled to high throughput, $\mathrm{Y} 2 \mathrm{H}$ and $\mathrm{RY} 2 \mathrm{H}$ generate a huge quantity of positive clones that need to be sequenced in order to identify binding partners and IDAs, respectively. Usually, each positive clone is sequenced and the user gets the results back in one or several fast-all (FASTA) sequence files. When working with large datasets, this step can generate hundreds of such files with the obvious consequence of significantly slowing down the identification of the bona fide protein partners. Furthermore, the sequencing product contains part of the cloning vector and a specific primer tag, which might severely limit the efficiency of alignment of clone DNA or protein sequence to public databases via the NCBI Basic Alignment Search Tool (Blast!) for identification (Altschul et al. 1990). An insertion or deletion can also occur during the cloning process, which will disrupt the open reading frame (ORF), resulting into incorrect identification. Finally, conventional sequencing only works well for up to roughly 500-800 bp (Mavromatis et al. 2012). Past that, chromatography signals degrade, thus sequencing becomes less reliable. Therefore, DNA fragments encoding for polypeptides larger than 200 amino acids cannot be easily sequenced. For RY2H, full length sequencing of such preys is particularly desirable to map the full complement of residue mutations that abrogate a bait-prey interaction. This issue is usually addressed by sequencing both strands using forward and reverse primers. However, this infers the subsequent assembly of both sequences and finding the right reading frame in the Cterminus, which is not trivial when dealing with large numbers of positive clones.

Finally, in the case of RY2H, spotting and summarizing bait mutations along the protein primary sequence in order to identify a potential surface of interaction for a given interacting partner requires a dedicated tool. To this end, we developed 2HybridTools, a handy software which simplify and speed up these steps by opening several sequencing FASTA files at the same time, by deleting unnecessary vector DNA, by optionally finding the right reading frame, and, in the case of paired-end sequencing, by overlapping corresponding sequencing fragments while trimming low-quality sequencing and keeping the resulting protein in frame, which is then protein-translated and identified via Blast. Additionally, 2HybridTools detects and summarizes mutations in a graphical report, and those mutations can be superimposed onto a protein model sequence if desired. We provide this tool for the users of two-hybrid technology, since there is no freely available such software suite. A commercial suite does exist (DNADynamo). However, it does not support multiple sequences, neither assembly of sequencing products using forward and reverse sequencing. While Contig Assembly Program 3 (CAP3), a DNA assembly program, can 
122 be used for this purpose since it supports these features (Huang \& Madan 1999), it is however

123

124

125

126

127

128

129

130

131

132

133

134

135

136

137

138

139

140

141

142

143

144

145

146

147

148

149

150

151

152

153

154

155

156

157

158

159

160

161

not dedicated to $\mathrm{Y} 2 \mathrm{H}$ and thus lacks features such as identification of clones or trimming of vector sequences. 2HybridTools also improves these two programs by introducing a model sequence against which clones are aligned, thus producing a summary of specific mutations resulting for example from a $\mathrm{RY} 2 \mathrm{H}$ screen, as well as open reading frame detection and automated Blast identification of clones. The efficiency of the program is illustrated here with two examples which we walk through, one from a regular $\mathrm{Y} 2 \mathrm{H}$ assay and another to analyze mutations from a RY2H assay.

\section{Methods}

\section{Availability}

2HybridTools is a portable Java application which requires BioJava (Holland et al. 2008) $\geq 1.5$, which is distributed with 2 HybridTools and installed in the application's system classpath, i.e. the $l i b$ directory or in the lib/ext directory of the Java runtime environment (JRE) virtual machine folder. Alternatively, BioJava is available at https://biojava.org/. 2HybridTools requires Java $\geq$ 1.5. The application is entirely open source. Binaries and example files for $\mathrm{Y} 2 \mathrm{H}$ as well as RY2H can be obtained freely at GitHub under https://github.com/pc297/2HybridTools.

\section{Algorithm flowchart}

An overview of the main steps behind the algorithmic logic used in 2HybridTools is shown as a diagram on Figure 1. The default mode of processing is $\mathrm{Y} 2 \mathrm{H}$, with no model input. Loading a sequence model enables the RY2H mode.

\section{Main interface}

The main interface, a JFrame object, can be viewed in Figure $\mathbf{2 A , B}$, where original sequences are shown (top text pane, a JTextPane object with JScrollBar) and colored according to their sequence nature (i.e. vector, coding sequence, low quality sequence), and processed sequences are aligned with amino acids residues colored according to their physicochemical properties (bottom text pane, a JTextPane object with JScrollBar). Multiple sequence files which can be loaded or appended to the current project (Figure 2A,B). DNA sequences are translated into proteins which are aligned and overlapping sequences are then displayed in comparison to sequence model. A File menu allows saving open reading frame analysis, fragment overlapping, translation, Blast and model alignment in either DNA or protein FASTA file formats. Mutation reports can also be saved from the Summary option once alignment to model has been carried out. Multiple options are available 1) vector sequence options ( $\mathrm{N}$ - and $\mathrm{C}$ - Terminus), 2) minimum protein or DNA overlapping size upon $\mathrm{N}$ - and $\mathrm{C}$-Terminal sequencing, 3) N-cutoff, i.e. accepted number of unidentified bases before discarding the 3' remainder of sequences, 4) proxy options for users requiring a proxy for Internet connection (Figure S1).

\section{Sequencing quality trimming}


162 The very start of sequencing files can contain a number of unidentified bases $(\mathrm{N})$ due to poor

163

164

165

166

167

168

169

170

171

172

173

174

175

176

177

178

179

180

181

182

183

184

185

186

187

188

189

190

191

192

193

194

195

196

197

198

199

200

201

sequencing quality in the first 15-40 bases at the 5' end of the fragments. Therefore only the nucleotide sequence following the first $\mathrm{N}$ will be used by the program as a default value. Likewise, bases following the second $\mathrm{N}$ at the 3 ' end will not be used neither. However, if required, the user can modify this default setting (Figure S1), which brings up a JOptionPane object.

\section{Vector trimming}

The default $\mathrm{N}$ - and $\mathrm{C}$-Terminus vector tag protein sequences are respectively STHAS and DPAFL, which are tags from pAD vector (Invitrogen ${ }^{\mathrm{TM}}$ ). These sequences can also be entered manually, either as DNA or amino-acid sequences and their position within the sequence will be identified by searching the entire translated sequences in all six open reading frames. This option allows choosing the number of bases in which the protein is in frame downstream of the vector sequence. Therefore, all bases prior to the start of the protein sequence are discarded (Figure $2 \mathbf{A}$, S1). To account for possible sequencing errors, mapping of vector sequences can be performed with one mismatch, which will be automatically allowed should the tag sequence not be matched in the prey sequences (Figure 1). This is achieved by catching a StringIndexOutOfBounds exception when not returning the index of a vector tag sequence in the prey sequence, with a loop going through the entire sequence, matching, in turn, each character of the query, then the next one, until the end of the vector string, with a counter that counts the number of times a character from the query was missed; queries resulting in a counter less than the number of set mismatches are retained. Since the vector sequences are at the beginning of the prey sequences (both for forward and reverse sequencing), we determine those be the first match with a mismatch in the sequence. These options are set via a custom JFrame object with input fields and sliders for text and distance to ORF, respectively.

\section{ORF analysis}

When this option is selected, the longest average ORF size will be determined in all sequences. Each sequence is translated in all six ORFs in the selected genetic code. This option can be useful if the fusion protein contains a Methionine residue in proximity of the insertion site to correctly identify the frame the insert is in. The strand and frame yielding the highest average translated size will thus be retained. Choosing this option will increase BLAST alignment scores however it should not be used when dealing with fusion proteins. When used, ORF information is added to the sequence identifier.

\section{Protein translation}

By default, the universal genetic code is selected. This can however be overridden for use with bacterial two-hybrid, in which case GUG and UUG start codons are documented (Kozak 1983). ORF and strand are user-defined except in the case of automatic ORF analysis in which these parameters are set up automatically. We made use of the BioJava translation method with the

Peer] reviewing PDF | (2019:01:34100:1:1:CHECK 27 May 2019) 
202 following exception: in the case of a codon containing an N, if the two other bases

203

204

205

206

207

208

209

210

211

212

213

214

215

216

217

218

219

220

221

222

223

224

225

226

227

228

229

230

231

232

233

234

235

236

237

238

239

240

241

unambiguously point towards a specific amino acid, e.g. GTN for Valine, then it will be translated as such. Otherwise, the result will be designated as $\mathrm{X}$ but will however not be treated as a mutation.

\section{Clone identification}

HTTP POST requests containing each translated sequence are directed to an Expert Protein Analysis System (ExPASy) (Appel et al. 1994) protein Blast service mirror using plain HTML. HTTP connections are maintained until each query is completed, in which case the HTTP response is parsed and the protein information subsequently retrieved (Figure 2A). This step is carried out by parsing an InputStreamReader object for hits, with the sequence query written to an OutputStreamWriter object. If no hit was found, this will be reported in the main user interface. Information corresponding to the best hit is added to the sequence identifier. To allow grouping in case of multiple prey proteins, Blast! results can be sorted alphabetically by checking the Sort Blast output. Sorting is performed using a Comparator class on BLAST hit information parsed from sequence identifiers.

\section{Sequence overlap for the assembly of forward and reverse sequencing products}

Sequences from the same clone are normally labeled similarly, with the name of the primer added, in our example, the $\mathrm{N}$-terminus sequence is labeled $\mathrm{AD}$ and the $\mathrm{C}$-terminus labeled "TERM" because we used primers AD and TERM respectively. If an overlap is found between two sequences with the specified minimum overlap, the resulting sequence will be labeled "ADTERM OVERLAPPED" (Figure 2B). The minimum overlap is by default set to 6 amino acid residues or $18 \mathrm{bp}$, corresponding to the minimum number of bases in a complete codon sequence that will be unique in the human genome (16 would be the minimum number of bases however that would yield an incomplete codon). If a corresponding N-terminus is not found for a given Cterminus, the latter will be labeled "TERM NON-OVERLAPPED". If a C-terminus is not found, neither translation nor overlapping alignment is attempted and the sequence is discarded. The Nterminus is then reverse-complemented from its vector tag onwards. We then use a local pairwise Smith-Waterman alignment (Smith \& Waterman 1981) at the amino acid or DNA level with high substitution, insertion, deletion and gap penalties, using an International Union of Pure and Applied Chemistry (IUPAC) identity substitution matrix. The largest possible alignment with the best score is used in order to overlap N- and C-termini. A minimum alignment length of 5 perfect matches is required for $\mathrm{N}$ - and $\mathrm{C}$ - terminus overlap, although this can be set as a parameter. This step is carried out by calling a SmithWaterman object from BioJava.

\section{Sequence model alignment and mutation report}

For RY2H, a DNA or protein model sequence can be loaded prior to analysis, which will result in all translated sequences being aligned in turn to the model protein sequence. The model can 
242

243

244

245

246

247

248

249

250

251

252

253

254

255

256

257

258

259

260

261

262

263

264

265

266

267

268

269

270

271

272

273

274

275

276

277

278

279

280

281

either be loaded from a local fasta file, or downloaded directly from NCBI by inputting the protein accession number. This is achieved by posting a URL containing the parsed NCBI accession number, eliciting a response in plain fasta text format. Selecting a model following translation will not cause sequences to be translated or overlapped again. We perform a SmithWaterman alignment (by calling a SmithWaterman object from BioJava) with low substitution penalties and high insertion, deletion and gap penalties using the BLOcks SUbstitution Matrix clustered at the 62\% level (BLOSUM62) substitution matrix (Henikoff \& Henikoff 1992). Nonoverlapped C-terminal fragments are also aligned. Only alignment scores greater than the specified or default alignment score, which corresponds to $5 \mathrm{x}$ model sequence length $\mathrm{x} 80 \%$, are kept. Sequences with alignment scores satisfying these parameters are maintained in the lower text pane (Figure 2B). To limit the rate of false positive discovery, including frame shift and termination mutations, sequences shorter or longer than the sequence model are discarded when retrieving mutations within an alignment. Results are then summarized above the sequence of reference with the precise mutation nature and counts and can be viewed by clicking the Summary button (Figure 3A,B). Finally, another graphical, color-coded overview of the mutation distribution along the protein can be obtained by pressing the Graph button (Figure 3C).

\section{Comparison to other software}

As mentioned above, other solutions that encompass sequence assembly already exist, and can thus be readily used for $\mathrm{Y} 2 \mathrm{H}$ analysis. A summary of the functionalities of 2 HybridTools compared to other similar programs is shown on Table 1. CAP3 (Huang \& Madan 1999), a recognized all-purpose sequence re-assembly program and one of the most widely used tool, can be used for the purpose of clone re-construction as it implements sequencing quality cut-off, clone identification using the latter. But it cannot assemble sequence(s) containing vector sequences. CAP3 was not written specifically for the purpose of $\mathrm{Y} 2 \mathrm{H} / \mathrm{RY} 2 \mathrm{H}$ analysis. Conversely, 2HybridTools features vector-trimming and multiple sequence Blast, which greatly facilitates clone identification. Moreover, CAP3 does not implement protein translation, which renders protein Blast impossible and thus would incorrectly align a DNA sequence containing a deletion induced by induced or spontaneous mutagenesis to a reference sequence. DNADynamo, a commercial software suite, also supports several of the above mentioned functions but does not support multiple sequences. Furthermore, 2HybridTools provides a unique, RY2H-dedicated mutation report function, which allows pinpointing mutations that could define a surface of interaction that plays an important role in protein-protein interactions. Overall, implementation of the automated Blast, vector trimming and translation features provided in 2HybridTools allow easier clone identification compare to CAP3, and provides a unique, RY2H-dedicated mutation report function, which is absent in DNADynamo. This feature thus allows an in-depth $\mathrm{Y} 2 \mathrm{H} / \mathrm{RY} 2 \mathrm{H}$ analysis, as detailed below.

\section{Results}


282

283

284

285

286

287

288

289

290

291

292

293

294

295

296

297

298

299

300

301

302

303

304

305

306

307

308

309

310

311

312

313

314

315

316

317

318

319

320

321

\section{SP1 Y2H Screening}

A yeast two-hybrid screening of the transcription factor SP1 (Kadonaga et al. 1987) was performed at the two-hybrid platform, CRCM (Inserm UMR891, Marseille, France) using a previously described protocol (Walhout \& Vidal 2001). The screen was performed using a human colon cDNA library, in order to discover potential new partners for SP1 involved in apoptosis (Vicart et al. 2006). We read the resulting FASTA sequencing files corresponding to positive clones ("SP1_2hybrid_screening.seq") into 2HybridTools using the File/Open Sequence(s) drop-down menu (Figure S3A). For this particular experiment, the vector tag sequence was STHAS, which we set in the Vector/Define 5' Vector menu (Figure S3B), with all other options left unchanged from their default values. ORF analysis in 2HybridTools, performed by pressing the Find ORFs button, correctly identified the first codon encoded by the prey and the right reading frame in all the clones isolated from the screen. This identification was necessary since several clones encoding the same partner were identified but they were not necessarily starting at the same amino acids within the protein. This is due to the cloning strategy used to create the cDNA library. To translate cDNA into protein and to trim vector sequences, as well as low sequencing quality sequencing ends, we hit the Go button, updating the main interface with the resulting trimmed sequences and translated sequences (Figure $\mathbf{2 A}$, top and bottom text fields). We then set out to identify the protein sequences of positive clones using the built-in automated Blast! feature, pressing the Blast results button in the main interface. This analysis revealed that the proteins CDX1 and RPL23 were overrepresented in the sequencing results (Figure 2A), yielding respectively 20 and 3 hits each. These proteins are thus likely partners of SP1. SP1 is known to interact with homeobox proteins (Park et al. 2007) and furthermore $C D X 1$ is a direct transcriptional target of SP1 (Gilmour et al. 2014; Lim \& Chang 2009). Interaction with RPL23 suggests that this interaction is cytoplasmic and could involve the synthesis or transport processes of the SP1 protein.

\section{Nsp10-Nsp16 Reverse Y2H Screening}

We have also performed a RY2H screening using the SARS non-structural proteins (Nsp) Nsp10 and nsp16 (Imbert et al. 2008; Lugari et al. 2010). Nsp10 was used as the bait to identify interaction-defective alleles (IDAs) of Nsp16. To this end, we generated a library of randomly mutated full-length Nsp16 that was screened against Nsp10 (Lugari et al. 2010). The Nsp16 cDNA being $897 \mathrm{pb}$ long, we sequenced positive clones (whereby the interaction between the bait and prey is abrogated) from both ends. This cDNA length allows forward and reverse sequencing products to overlap. As with conventional $\mathrm{Y} 2 \mathrm{H}$, we input the resulting FASTA sequencing files (MultiFastaNsp16 mutants.seq, containing both forward and reverse sequencing products) using the File/Open Sequence(s) menu (Figure S3C). We also read the Nsp16 reference cDNA sequence (NSP16DNAmodel.txt) using the File/Load Model (Optional) menu (or, alternatively, as a protein sequence, using the file NSP16PROTEINmodel.txt) (Figure S3C). For this experiment, we set the vector tag sequence as STHAS and the field "Distance to ORF", the distance of the vector tag sequence, to the ORF as 6 amino acids (Vector/Define 5' Vector) 
322 (Figure S3D, top). Since positive clones were sequenced from both ends, we also input a 3'

323

324

325

326

327

328

329

330

331

332

333

334

335

336

337

338

339

340

341

342

343

344

345

346

347

348

349

350

351

352

353

354

355

356

357

358

359

360

vector tag sequence as DPAFL (Vector/Define 3' Vector), leaving the field Distance to ORF to 0 as the ORF ends directly upstream of this sequence (Figure S3D, bottom). We set all other parameters to their default values. We then hit the Go button in the main interface, starting processing of sequences, overlapping of sequencing ends, and aligning to the reference sequence, updating the main display with trimmed sequences (Figure 2B). This analysis allowed reconstructing 91 Nsp16 full-length proteins (from 182 DNA sequences) out of 189 original Nand C-Terminus cDNA sequences (Figure 2B). Only 7 sequences were not overlapping, of which 4 correspond to non-matched N-Terminus fragments. To obtain a detailed per-residue summary of mutations in the cDNA sequences corresponding to positive prey proteins, we hit the Summary button in the main interface. A detailed view of the mutations on the first 100 residues of Nsp16 that led to lost Nsp10-Nsp16 is shown on Figure 3A,B. To bring up a heatmap view of mutations, we hit the Graph button in the main interface. A summary of the residue mutation frequencies on the entire Nsp16 protein is shown on Figure 3C.

\section{Discussion}

2HybridTools improves on other non-dedicated software used for $\mathrm{Y} 2 \mathrm{H}$ analysis software by combining features uniquely supported by some in an all-in-one fashion. To our knowledge, our software is the first to implement dedicated $\mathrm{RY} 2 \mathrm{H}$ analysis. The alignment to a reference sequence as well as the identification of mutation positions, nature and frequencies provides a valuable tool for RY2H. Ensuring that positive sequences, which resulted in abolishing the bait/prey interaction, were in frame also allows dismissing false positives. The ability to align sequencing products from both ends when dealing with sequences longer 500-800 bps in an automated fashion, for multiple sequences, also provides a net gain of analysis time for $\mathrm{Y} 2 \mathrm{H}$ and $\mathrm{RY} 2 \mathrm{H}$ end-users, as does the automated identification of multiple clones. Thanks to its Java implementation, 2HybridTools is also platform-independent and thus easily accessible to $\mathrm{Y} 2 \mathrm{H} / \mathrm{RY} 2 \mathrm{H}$ users. The ability to identify specific mutations from RY2H also provides the basis for focused study of interaction residues. Although only more direct protein-protein interaction assays such as glutathione S-transferase (GST) pull-down, bioluminescence or fluorescence resonance energy transfer (BRET or FRET) (Lohse et al. 2012) will yield definitive results as was done for Nsp10 and Nsp14 (Bouvet et al. 2014), the combined use of this tool and 3D structures is a good indicator as to where to start looking using these high-resolution techniques.

\section{Conclusions}

A possible future development for this tool would be the automated mapping of mutations using 3D modeling software such as Rasmol and automatically generated Rasmol scripts, which would directly map mutations on a given crystal structure. In fine, our software can be used to expand current protein-protein interaction databases. Finally, as recent developments to Y2H/RY2H have been made to exploit next-generation sequencing (NGS) to sequence positive clones (Suter

Peerj reviewing PDF | (2019:01:34100:1:1:CHECK 27 May 2019) 
361 et al. 2015), a further development to this software would entail the use of NGS sequencing 362 results.

363

364

365

366

367

368

369

370

371

372

373

374

375

376

377

378

379

380

381

382

383

384

385

386

387

388

389

390

391

392

393

394

395

396

397

398

399

400

\section{Acknowledgements}

We express our special thanks to Frédéric Rosa and Virginia Summerour for original testing.

\section{References}

Altschul SF, Gish W, Miller W, Myers EW, and Lipman DJ. 1990. Basic local alignment search tool. J Mol Biol 215:403-410. 10.1006/jmbi.1990.9999

S0022283680799990 [pii]

Appel RD, Bairoch A, and Hochstrasser DF. 1994. A new generation of information retrieval tools for biologists: the example of the ExPASy WWW server. Trends Biochem Sci 19:258-260.

Bouvet M, Lugari A, Posthuma CC, Zevenhoven JC, Bernard S, Betzi S, Imbert I, Canard B, Guillemot JC, Lecine P, Pfefferle S, Drosten C, Snijder EJ, Decroly E, and Morelli X. 2014. Coronavirus Nsp10, a critical co-factor for activation of multiple replicative enzymes. J Biol Chem 289:25783-25796. 10.1074/jbc.M114.577353

M114.577353 [pii]

Bruckner A, Polge C, Lentze N, Auerbach D, and Schlattner U. 2009. Yeast two-hybrid, a powerful tool for systems biology. Int J Mol Sci 10:2763-2788. 10.3390/ijms10062763

Fields S, and Song O. 1989. A novel genetic system to detect protein-protein interactions. Nature 340:245-246. 10.1038/340245a0

Gilmour J, Assi SA, Jaegle U, Kulu D, van de Werken H, Clarke D, Westhead DR, Philipsen S, and Bonifer C. 2014. A crucial role for the ubiquitously expressed transcription factor Sp1 at early stages of hematopoietic specification. Development 141:2391-2401. 10.1242/dev.106054

dev.106054 [pii]

Hamdi A, and Colas P. 2012. Yeast two-hybrid methods and their applications in drug discovery. Trends Pharmacol Sci 33:109-118. 10.1016/j.tips.2011.10.008

Henikoff S, and Henikoff JG. 1992. Amino acid substitution matrices from protein blocks. Proc Natl Acad Sci U S A 89:10915-10919.

Holland RC, Down TA, Pocock M, Prlic A, Huen D, James K, Foisy S, Drager A, Yates A, Heuer M, and Schreiber MJ. 2008. BioJava: an open-source framework for bioinformatics. Bioinformatics 24:2096-2097. btn397 [pii]

10.1093/bioinformatics/btn397

Hollingsworth R. 2004. Target discovery using the yeast two-hybrid system. Drug discovery today : Targets 3:97-103.

Huang X, and Madan A. 1999. CAP3: A DNA sequence assembly program. Genome Res 9:868877.

Hughes TR. 2002. Yeast and drug discovery. Funct Integr Genomics 2:199-211. 
401

402

403

404

405

406

407

408

409

410

411

412

413

414

415

416

417

418

419

420

421

422

423

424

425

426

427

428

429

430

431

432

433

434

435

436

437

438

439

440

Imbert I, Snijder EJ, Dimitrova M, Guillemot JC, Lecine P, and Canard B. 2008. The SARSCoronavirus PLnc domain of nsp3 as a replication/transcription scaffolding protein. Virus Res 133:136-148. S0168-1702(07)00447-9 [pii]

10.1016/j.virusres.2007.11.017

James P, Halladay J, and Craig EA. 1996. Genomic libraries and a host strain designed for highly efficient two-hybrid selection in yeast. Genetics 144:1425-1436.

Kadonaga JT, Carner KR, Masiarz FR, and Tjian R. 1987. Isolation of cDNA encoding transcription factor $\mathrm{Sp} 1$ and functional analysis of the DNA binding domain. Cell 51:1079-1090. 0092-8674(87)90594-0 [pii]

Kozak M. 1983. Comparison of initiation of protein synthesis in procaryotes, eucaryotes, and organelles. Microbiol Rev 47:1-45.

Lim J, Hao T, Shaw C, Patel AJ, Szabo G, Rual JF, Fisk CJ, Li N, Smolyar A, Hill DE, Barabasi AL, Vidal M, and Zoghbi HY. 2006. A protein-protein interaction network for human inherited ataxias and disorders of Purkinje cell degeneration. Cell 125:801-814.

Lim K, and Chang HI. 2009. O-GlcNAc modification of Sp1 inhibits the functional interaction between Sp1 and Oct1. FEBS Lett 583:512-520. 10.1016/j.febslet.2008.12.007

S0014-5793(08)00992-7 [pii]

Lohse MJ, Nuber S, and Hoffmann C. 2012. Fluorescence/bioluminescence resonance energy transfer techniques to study G-protein-coupled receptor activation and signaling. Pharmacol Rev 64:299-336. 10.1124/pr.110.004309

pr.110.004309 [pii]

Lugari A, Betzi S, Decroly E, Bonnaud E, Hermant A, Guillemot JC, Debarnot C, Borg JP, Bouvet M, Canard B, Morelli X, and Lecine P. 2010. Molecular mapping of the RNA Cap 2'-O-methyltransferase activation interface between severe acute respiratory syndrome coronavirus nsp10 and nsp16. J Biol Chem 285:33230-33241. 10.1074/jbc.M110.120014

M110.120014 [pii]

Mavromatis K, Land ML, Brettin TS, Quest DJ, Copeland A, Clum A, Goodwin L, Woyke T, Lapidus A, Klenk HP, Cottingham RW, and Kyrpides NC. 2012. The fast changing landscape of sequencing technologies and their impact on microbial genome assemblies and annotation. PLoS One 7:e48837. 10.1371/journal.pone.0048837

Park SY, Kim JB, and Han YM. 2007. REST is a key regulator in brain-specific homeobox gene expression during neuronal differentiation. J Neurochem 103:2565-2574. 10.1111/j.14714159.2007.04947.x

JNC4947 [pii]

Parrish JR, Gulyas KD, and Finley RL, Jr. 2006. Yeast two-hybrid contributions to interactome mapping. Curr Opin Biotechnol 17:387-393. 10.1016/j.copbio.2006.06.006

Rual JF, Venkatesan K, Hao T, Hirozane-Kishikawa T, Dricot A, Li N, Berriz GF, Gibbons FD, Dreze M, Ayivi-Guedehoussou N, Klitgord N, Simon C, Boxem M, Milstein S, Rosenberg J, Goldberg DS, Zhang LV, Wong SL, Franklin G, Li S, Albala JS, Lim J, 
441

442

443

444

445

446

447

448

449

450

451

452

453

454

455

456

457

458

459

460

461

462

463

464

465

466

467

468

469

470

471

472

473

474

475

476

477

478

479

480

Fraughton C, Llamosas E, Cevik S, Bex C, Lamesch P, Sikorski RS, Vandenhaute J, Zoghbi HY, Smolyar A, Bosak S, Sequerra R, Doucette-Stamm L, Cusick ME, Hill DE, Roth FP, and Vidal M. 2005. Towards a proteome-scale map of the human proteinprotein interaction network. Nature 437:1173-1178.

Sardina DS, Micale G, Ferro A, Pulvirenti A, and Giugno R. 2018. INBIA: a boosting methodology for proteomic network inference. BMC Bioinformatics 19:188. 10.1186/s12859-018-2183-5

Smith TF, and Waterman MS. 1981. Identification of common molecular subsequences. J Mol Biol 147:195-197. 0022-2836(81)90087-5 [pii]

Suter B, Zhang X, Pesce CG, Mendelsohn AR, Dinesh-Kumar SP, and Mao JH. 2015. NextGeneration Sequencing for Binary Protein-Protein Interactions. Front Genet 6:346. 10.3389/fgene. 2015.00346

Vicart A, Lefebvre T, Imbert J, Fernandez A, and Kahn-Perles B. 2006. Increased chromatin association of Sp1 in interphase cells by PP2A-mediated dephosphorylations. J Mol Biol 364:897-908. S0022-2836(06)01227-7 [pii]

10.1016/j.jmb.2006.09.036

Vidal M, and Legrain P. 1999. Yeast forward and reverse 'n'-hybrid systems. Nucleic Acids Res 27:919-929.

Walhout AJ, and Vidal M. 2001. High-throughput yeast two-hybrid assays for large-scale protein interaction mapping. Methods 24:297-306. 10.1006/meth.2001.1190

S1046-2023(01)91190-9 [pii]

\section{Table and figure legends}

Table 1. Features supported by 2 HybridTools compared to available programs used for $\mathrm{Y} 2 \mathrm{H}$.

Figure 1. Algorithmic flowchart of the implementation of 2HybridTools. The flow of information is represented by arrows. Parallelograms represent inputs, rounded rectangles events, straight rectangles processing steps, and diamonds represent conditions. Fields in bold represent mandatory steps.

Figure 2. Main interface of $2 \mathrm{HybridTools}$ in $\mathrm{Y} 2 \mathrm{H}$ and $\mathrm{RY} 2 \mathrm{H}$ mode. (A) Functionalities specific to the $\mathrm{Y} 2 \mathrm{H}$ mode are highlighted in callout bubbles. These include support for multiple sequences, clone identification via protein Blast and ORF discovery. (B) Functionalities specific to the RY2H mode are highlighted in callout bubbles. These include comparison to a reference sequence, forward and reverse sequencing product assembly and mutation summary.

Figure 3. Mutation summaries and localization using a reference sequence in RY2H mode. (A,B) Mutation reports of Nsp16 clones disrupting interaction with Nsp16, 1-50 aa (A) and 51100 aa (B). (C) Graphical overview of mutation location on Nsp16, with the number of mutations indicated as a color-coded heatmap, from left to right. 
481

482 Supplementary figure legends

483 Figure S1. Options of 2HybridTools. Drop down menus showing (A) file options, (B) vector 484

485

486

487

488

489

490

491

492

493

494

495

496

options: 5' and 3' vector sequences, mismatches and (C) overlap options for assembly of forward and reverse sequencing products.

Figure S2. Optional sorting of Blast! output in 2HybridTools. Alphabetical sorting is enabled by the Sort Blast output checkbox.

Figure S3. Walkthrough of $\mathrm{Y} 2 \mathrm{H}$ and RY2H examples. (A, B) Y2H mode, loading sequences (A), and setting 5' vector tag sequence as STHAS, with ORF starting directly after tag sequence (B). (C,D) RY2H mode, loading sequences as well as reference model (C), and setting 5' vector tag sequence as STHAS, with ORF starting 6 residues after tag sequence (D, top); setting 3' vector tag as DPAFL, with ORF starting ending directly before tag sequence (D, bottom). 


\section{Figure 1}

Algorithmic flowchart of the implementation of 2 HybridTools.

The flow of information is represented by arrows. Parallelograms represent inputs, rounded rectangles events, straight rectangles processing steps, and diamonds represent conditions. Fields in bold represent mandatory steps. 


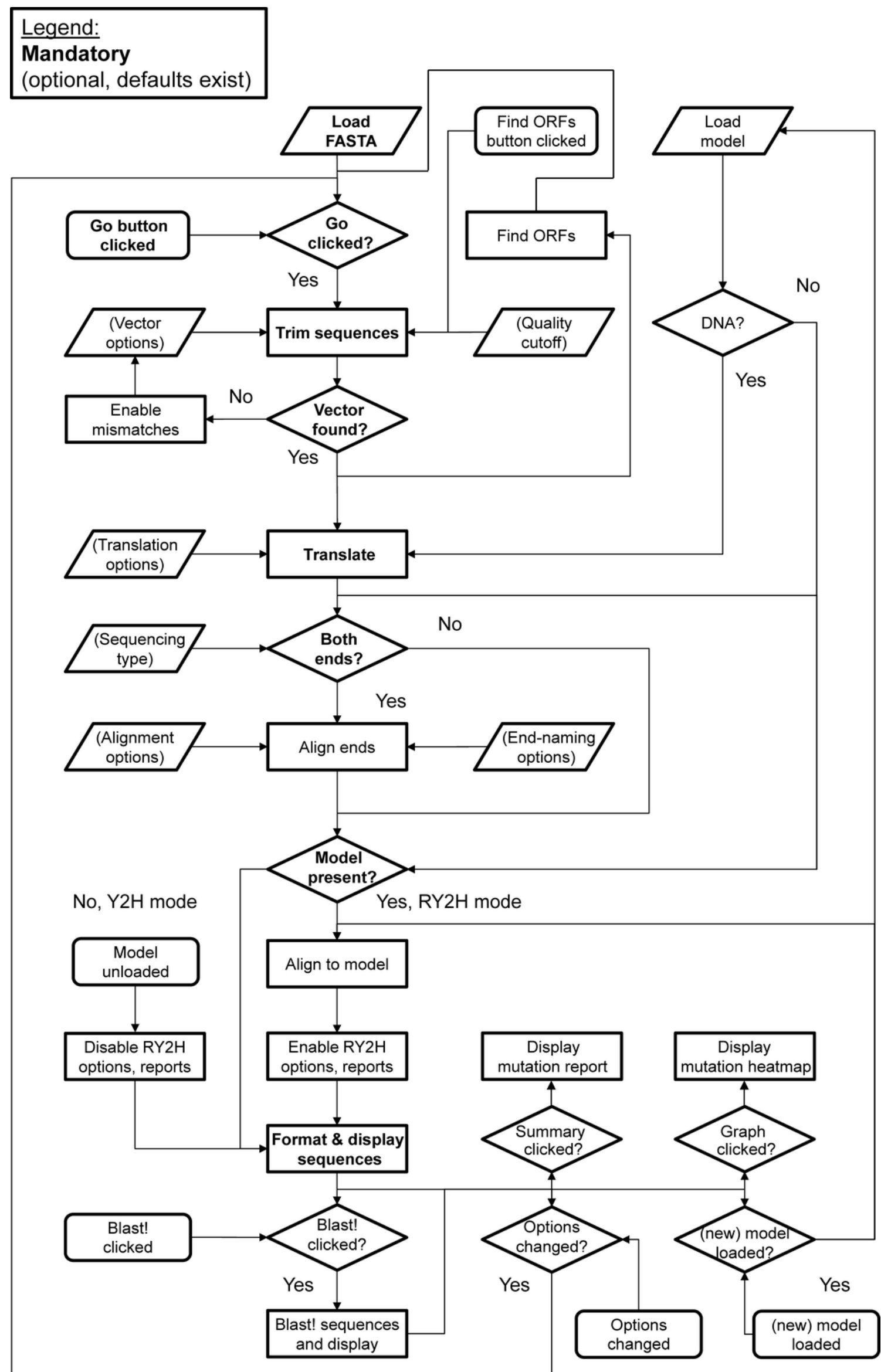


Figure 2

Main interface of $2 \mathrm{HybridTools}$ in $\mathrm{Y} 2 \mathrm{H}$ and $\mathrm{RY} 2 \mathrm{H}$ mode.

(A) Functionalities specific to the $\mathrm{Y} 2 \mathrm{H}$ mode are highlighted in callout bubbles. These include support for multiple sequences, clone identification via protein Blast and ORF discovery. (B) Functionalities specific to the RY2H mode are highlighted in callout bubbles. These include comparison to a reference sequence, forward and reverse sequencing product assembly and mutation summary.

A

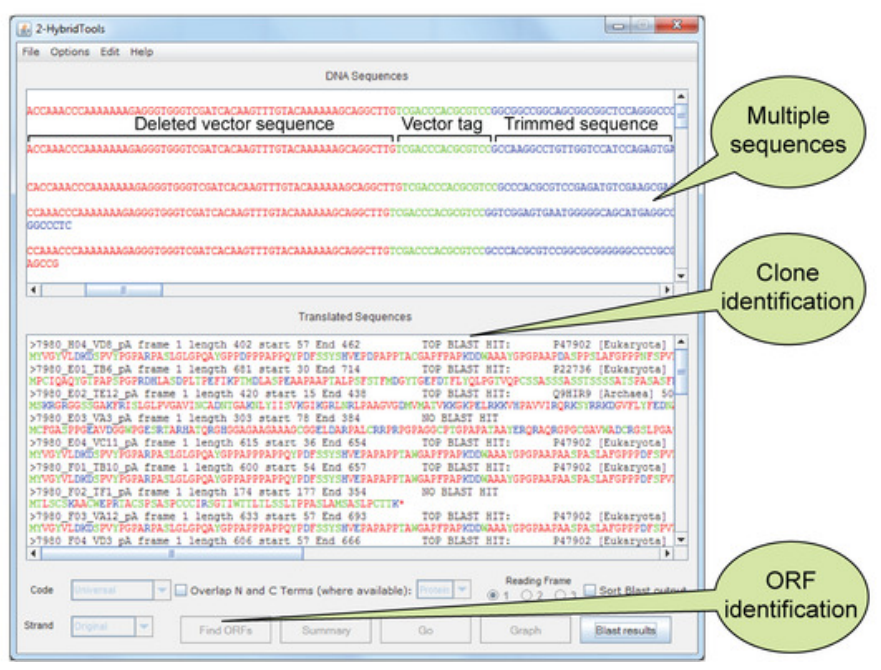

B

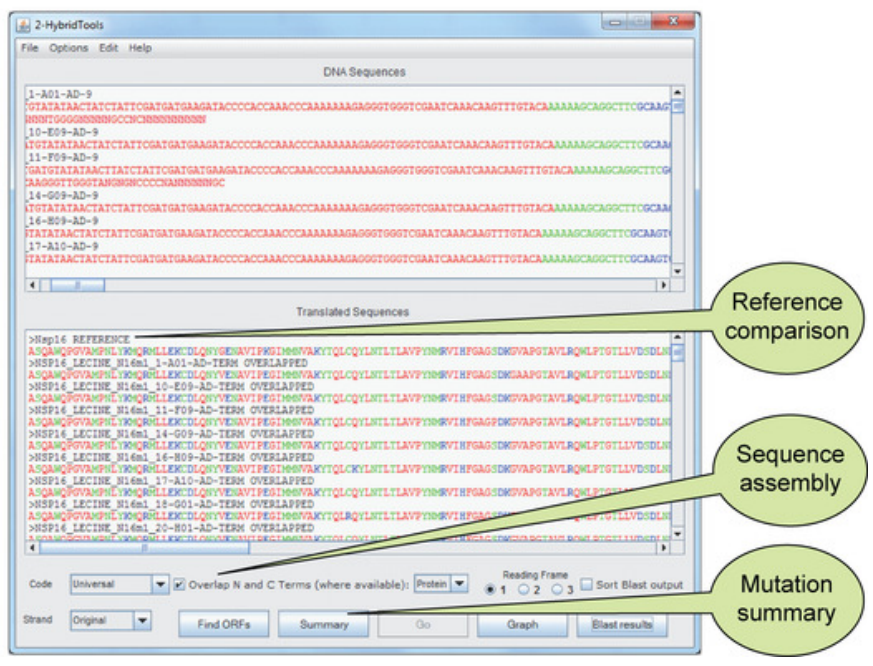


Figure 3

Mutation summaries and localization using a reference sequence in $\mathrm{RY} 2 \mathrm{H}$ mode.

(A,B) Mutation reports of Nsp16 clones disrupting interaction with Nsp16, 1-50 aa (A) and 51-100 aa (B). (C) Graphical overview of mutation location on Nsp16, with the number of mutations indicated as a color-coded heatmap, from left to right.

A

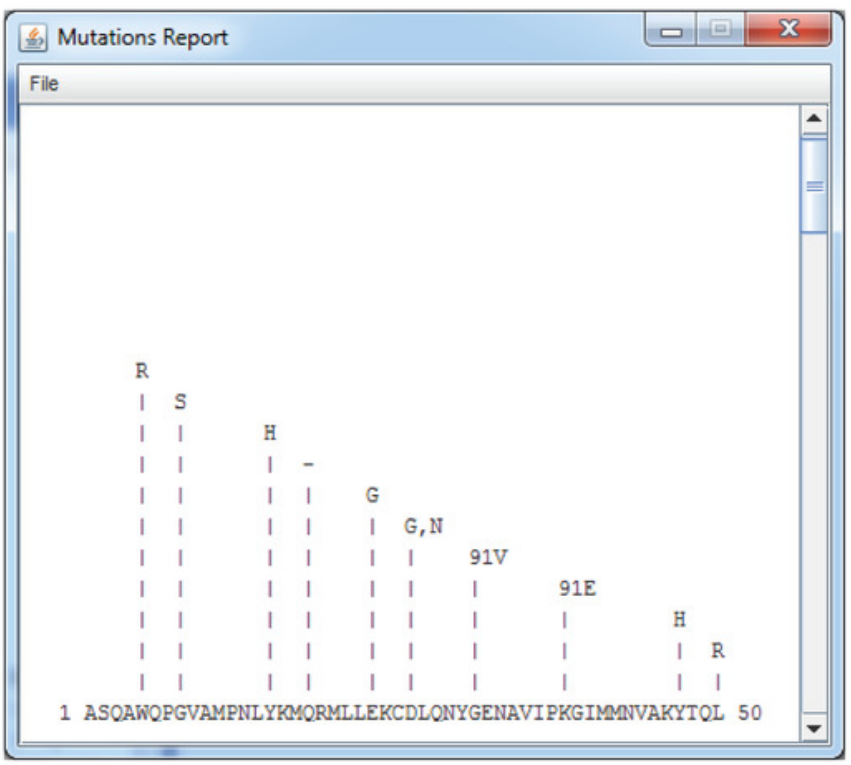

B

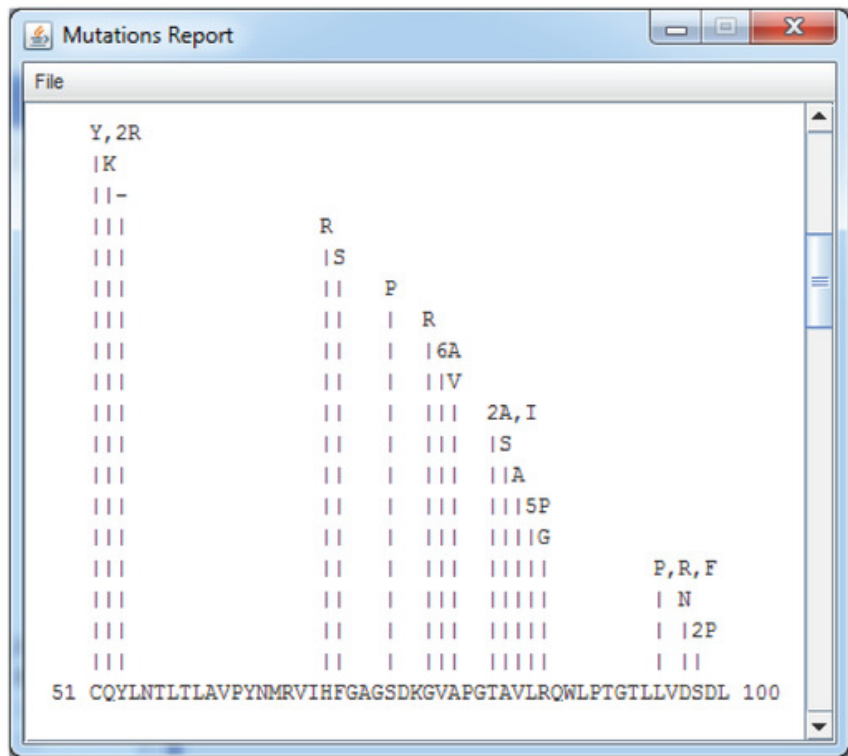

C

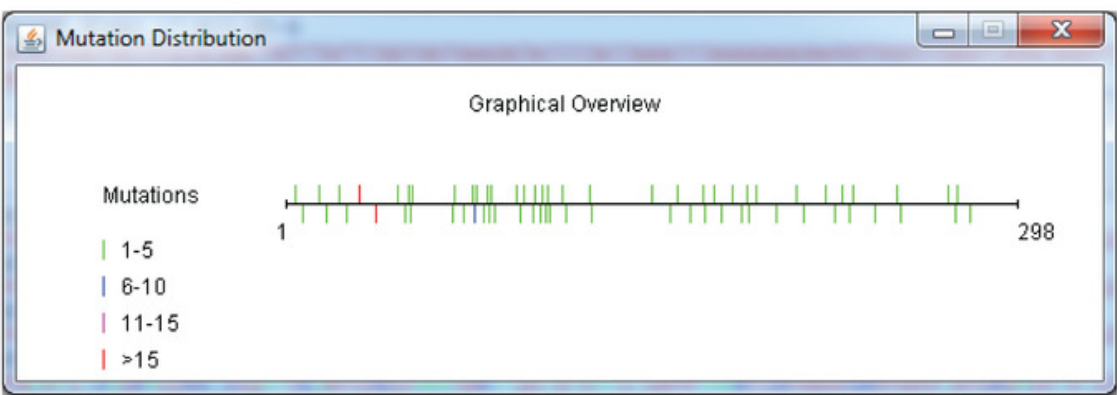




\section{Table 1 (on next page)}

Features supported by $2 \mathrm{HybridTools} \mathrm{compared} \mathrm{to} \mathrm{available} \mathrm{programs} \mathrm{used} \mathrm{for} \mathrm{Y} 2 \mathrm{H}$. 


\begin{tabular}{|c|c|c|c|}
\hline Features/Program & 2HybridTools & CAP3 & DNADynamo \\
\hline Assemble forward and reverse sequences & $\checkmark$ & $\checkmark$ & $\checkmark$ \\
\hline Multiple sequence support & $\checkmark$ & $\checkmark$ & x \\
\hline Vector trimming & $\checkmark$ & $\mathbf{x}$ & $\checkmark$ \\
\hline Vector library & $\mathbf{x}$ & $\mathbf{x}$ & $\checkmark$ \\
\hline Protein translation & $\checkmark$ & $\mathbf{x}$ & $\checkmark$ \\
\hline Mutation summary & $\checkmark$ & $\mathbf{x}$ & $\mathbf{x}$ \\
\hline Integrated Blast & $\checkmark$ & $\mathbf{x}$ & $\checkmark$ \\
\hline Open reading frame identification & $\checkmark$ & $\mathbf{x}$ & $\checkmark$ \\
\hline Map restriction sites & $\boldsymbol{x}$ & $\mathbf{x}$ & $\checkmark$ \\
\hline Free licence & $\checkmark$ & $\checkmark$ & $\mathbf{x}$ \\
\hline
\end{tabular}

1 